\title{
An analytical model for turbulence scattered rays in the shadow zone for outdoor sound propagation calculation
}

\author{
Yiu Wai Lam \\ Acoustics Research Centre, University of Salford, Salford M5 4WT, United Kingdom
}

(Received 26 June 2008; revised 2 December 2008; accepted 9 January 2009)

\begin{abstract}
In outdoor sound propagation, an inherent problem of the ray tracing method is its inability to determine the sound pressure level in the shadow zone, where geometrical rays do not penetrate. This is a serious problem in a turbulent atmosphere where significant sound energy will be scattered into the shadow. Empirical corrections that are determined from measurements or numerical simulations are limited to situations within the bounds of the empirical corrections. This paper describes a different approach where the ray tracing model is modified analytically into a scattered ray model. Rays are first diffracted from the shadow boundary, which is determined by the geometrical ray paths. The diffracted rays are then scattered by turbulence in their way to the receiver. The amount of scatter is determined from turbulence statistics that are determined from a Gaussian turbulence model. Most of the statistics are determined analytically except one element, which is determined empirically from numerical simulations. This turbulence scattered ray model is shown to have good accuracy against calculations based on the parabolic equation, and against previously published measurement data. It was found that the agreement is good both with and without turbulence, at distance up to $2 \mathrm{~km}$ from the shadow boundary.
\end{abstract}

(C) 2009 Acoustical Society of America. [DOI: 10.1121/1.3076928]

PACS number(s): 43.28.Gq, 43.28.Js, 43.50.Vt [JWP]

Pages: $1340-1350$

\section{INTRODUCTION}

The calculation of outdoor sound propagation is an important element in environmental noise assessments. Since the outdoor environment is complex, it is necessary for such calculations to take into account a large number of environmental factors, such as ground attenuation and atmospheric effects. A number of significant advancements have been made in the past few decades, and there now exist several accurate methods for calculation. ${ }^{1-3}$ The parabolic equation (PE) method is particularly useful for long range sound propagation because of its accuracy and its ability to take into account range dependent ground and atmospheric conditions. Recent advancements in accelerating the calculation ${ }^{4}$ also greatly enhanced its practical applicability. However, despite these advances, the method is still expensive to use at high frequencies, and is not readily usable in complex surroundings such as in urban cities where sharp changes in topography, meteorology, or material conditions frequently occur. For calculations in such surroundings, the ray tracing method $^{5}$ provides a more practical alternative approach. For example, in the current noise mapping exercise in Europe, most of the calculations are based on ray models.

Ray models in outdoor sound propagation come in different forms. ${ }^{6-9}$ All are based on geometrical considerations. The variations come from the different degrees of approximation they place on the wave behavior along the propagation path. The ray tube approach and the semi-analytical ray model take into account more of the wave behavior, but can suffer from singularity effects such as caustics. The heuristic ray approach and its variant, which only use the ray paths to provide information on the phase and wave coherence, are easier to use, and can provide acceptable accuracy for engi- neering calculations. However, these ray models suffer from the same inherent problem that they cannot determine the propagation of sound into the shadow zone. By nature of their geometrical assumption, it is not possible for rays to penetrate into the shadow. There are various suggestions to overcome this problem. If the atmosphere can be assumed to be non-turbulent, range independent, and has a linear vertical sound speed profile, then the residue series approach ${ }^{10}$ can be used to calculate the diffraction of sound into the shadow. However, this solution requires the determination of the roots of a complex function, which is difficult to obtain accurately deep in the shadow. Moreover, the inability to account for turbulence is a serious limitation for calculations involving real atmospheres. In realistic environments, a more practical approach is to apply empirical adjustments to the sound level in the shadow zone. For example, a recent suggestion ${ }^{11}$ uses corrections that are determined empirically from a large number of numerical simulations. However, purely empirical corrections are fundamentally limited by the conditions under which they are developed. In contrast, an analytical approach will be more flexible, and could provide a better understanding of the underlying problem.

This paper describes the development of an analytical approach based on ray tracing to calculate the propagation of sound into the shadow zone. The basic ray tracing model is based on a discretized implementation of the heuristic model. ${ }^{8}$ In an upward refracting case where a shadow is formed, the diffraction of sound into the shadow is assumed here to be analogous to the geometrical diffraction by an equivalent barrier in a homogeneous atmosphere. The scattering into the shadow zone due to turbulence is calculated 
from statistics that are determined from a Gaussian turbulence model of the atmosphere. The accuracy of the model will be tested against the PE using published cases in literature.

\section{THE RAY TRACING MODEL}

Our basic ray tracing is based largely on a numerical approach. Once launched, rays are traced individually through the atmosphere to find the trajectory of the ray that forms the shadow boundary. The overall sound speed profile in the atmosphere can be arbitrary. In our implementation, the atmosphere is divided into a grid of small rectangular cells. The cell division is chosen such that the change in the sound speed within each cell is small enough to be approximated by a linear profile, so that an analytical solution ${ }^{8}$ can be used within each cell to determine the ray propagation trajectory. The rule of thumb is to set the grid size at 1/10 of the length scale of the fastest changing condition. In practice, in the absence of turbulence, the grid size is typically $1 \mathrm{~m}$ in the vertical direction to accommodate the expected logarithmic shape of the sound speed profile near ground. The grid size in the horizontal direction is set at $5 \mathrm{~m}$ to accommodate likely range dependent variations of the terrain and meteorological conditions. However, when simulating the scattering of rays due to atmospheric turbulence, the grid size is set at $1 / 10$ of the smallest length scale of the turbulence structure. Since the Gaussian turbulence spectrum used in this paper has a length scale of $1.1 \mathrm{~m}$, the grid size used is $0.11 \mathrm{~m}$. The ground terrain, which can be undulating, determines the lower boundary of the computational grid. Once the reflection geometry is worked out by the numerical ray tracing, the effect of the ground at each reflection can then be approximated by the Weyl-van der Pol formulation. ${ }^{12,13}$ The height of the upper boundary of the computational grid is set using the rule of thumb of 1/10 of the maximum horizontal range.

In a downward refracting case where there are multiple rays passing through a sampling area, the effect of turbulence can be approximated by the reduction in coherence between the rays using the mutual coherence function. ${ }^{8}$ However, as is common in all geometrical ray tracing methods, the procedure will fail to predict the sound pressure in the shadow zone of an upward refracting atmosphere. By definition no geometrical rays can penetrate into the shadow. In literature, several solutions to this problem have been suggested. In the simple case of an atmosphere with a linear vertical sound speed profile and has no turbulence, the sound field can be represented by an analytical normal mode solution. The residue series solution ${ }^{10}$ can then be derived for an upward refracting case. However, this is not a practical solution in view of its limitations concerning the sound speed profile and turbulence. The comparisons published by L'Espérance et $a l .{ }^{8}$ show that it can substantially over-estimate the attenuation in real life situations where turbulence is present. For realistic atmospheric conditions that include turbulence, a common approach is to introduce empirical scattering to the ray trajectory ${ }^{9}$ or to use empirical estimates of the sound pressure. ${ }^{11}$ The dependence on empirical values significantly limits the applicability of such approaches. For example, in

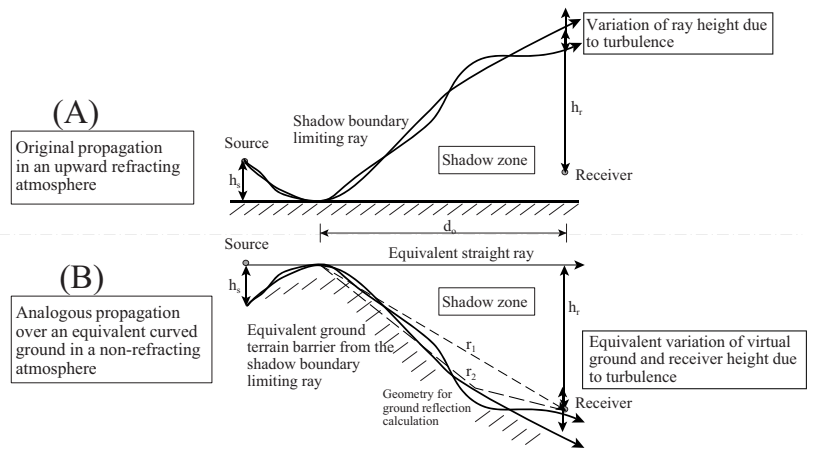

FIG. 1. Turbulence scattering of the analogous shadow boundary diffraction model. Upper half is the scattering of the original shadow boundary ray path over a flat ground. Lower half is the resulting scattering in the analogous model of a curved surface in a homogeneous atmosphere.

the ray model of Heimann and Gross, ${ }^{9}$ the effect of shadow diffraction is simulated by randomly changing the propagation direction of a ray when the ray is close to the ground, where diffraction is thought to be most important. Unfortunately, the physical mechanism for this ray diversion is not fully formulated. Instead, the implementation relies on empirical constants and assumed dependence on the height from the ground. Although the model worked well in some cases, ${ }^{9}$ it is doubtful that it will work equally well in situations outside the range of conditions under which the empirical parameters were derived without a full understanding of the physics involved. In this paper, we propose to address this problem by means of an analytical ray diffraction model.

\section{SHADOW BOUNDARY RAY DIFFRACTION MODEL}

In here, we use a shadow ray diffraction model that is based on the analogy between a curved ray path in the presence of refraction, and a curved surface in a homogeneous atmosphere in which there is no refraction. This analogy has been used successfully in studies involving linear sound speed profiles and analogous cylindrical surfaces. ${ }^{14}$ In here we apply the concept to an arbitrary sound speed profile and a generalized terrain with finite impedance cover. The analogy is illustrated in Fig. 1.

In the analogous virtual barrier configuration, the sound level at the receiver is calculated from the diffraction path from the barrier top to the receiver by applying simple thin barrier diffraction formulas. Here, one significant point to note is that the path difference cannot be calculated from the physical source location in the analogous barrier configuration. This is obvious if one considers the special case when the source is close to the barrier top (or the ground reflection point of the shadow boundary ray). An example is a strong upward refracting atmosphere where the shadow boundary is close to the source. In this case the path difference between the direct source to receiver path and the diffracted path over the barrier top is always small no matter how deep the receiver is in the shadow. This will give rise to only small attenuation even deep in the shadow. This is clearly incorrect. One would expect the attenuation to be higher with a stronger upward refracting atmosphere and a shadow boundary closer to the source. The explanation for this odd behav- 
ior is that the analogy is based on geometrical ray acoustics. Since a ray is, in fact, an approximation of a plane wave, the "equivalent straight ray" in the analogous virtual barrier configuration should be considered as a plane wave. The propagation should therefore be from a source that is far away. Hence the path difference should be calculated with the source placed at infinity, which is equivalent to calculating the difference between $r_{1}$ and $d_{0}$ in Fig. 1 .

In addition to the shadow boundary diffraction, the sound level at the receiver is also affected by attenuation due to ground reflection. This is clearly the case if one considers the sound level just before and after the shadow boundary. Before the boundary both direct and ground reflected rays are present and therefore the sound level will suffer ground attenuation, which can be as much as $20 \mathrm{~dB}$ at frequencies where ground attenuation is strongest. Just after the boundary, the shadowing (virtual barrier) effect is still small, and the attenuation will be of the order of only $5 \mathrm{~dB}$ (the usual line-of-sight barrier attenuation). Obviously, one would not expect the ground attenuation to just disappear when the receiver passes from one side to the other of the shadow boundary into the shadow. Hence it is necessary to correct for ground attenuation in the analogous virtual barrier model as well. As a first approximation, the ground attenuation is determined simply from the geometry of the geometrical reflection from the barrier top to the receiver. The local slope of the ground near the reflection point is used to correct for the ground reflection angle.

The prediction from this shadow boundary diffraction model is compared against a standard Crank-Nicolson $\mathrm{PE}^{15,16}$ prediction using the benchmark case $3 .^{17}$ This benchmark case has a flat ground with finite impedance. The atmosphere is strongly upward refracting with a linear sound speed profile of $-0.1 / \mathrm{s}$, but has no turbulence. The refraction is very strong. The shadow boundary at the receiver height is about $300 \mathrm{~m}$ from the source. The PE solution is a well established technique for outdoor sound propagation. ${ }^{17}$ It has been shown to have very good accuracy in the benchmark cases. ${ }^{17}$ It is therefore chosen to be the reference in this and subsequent comparisons. Figures 2(a), 2(b), and 2(c) show the comparisons at the frequencies of 10,100 , and $1000 \mathrm{~Hz}$, respectively. The $y$-axis is in terms of the transmission loss (TL) as defined in the benchmark paper. ${ }^{17}$ The lower limit of the $y$-axis is set at $-80 \mathrm{~dB}$ as is set in the benchmark paper. The horizontal distance is shown to be $10 \mathrm{~km}$, which covers a large range deep into the shadow. Also shown in the figures is the predicted sound pressure in free field in a still atmosphere (with air absorption). This is to allow the attenuation due to ground effect and atmospheric refraction alone to be easily identified. The figures show that the simple model proposed here for the shadow diffraction effect has good accuracy in this benchmark case. On the whole, the prediction agrees well with the prediction by the PE. The increases in the TL due to ground attenuation [most dominant at $100 \mathrm{~Hz}$ in Fig. 2(b)] and due to frequency [Fig. 2(c)] are well predicted. Given the simplicity of the shadow diffraction model, the agreement is quite remarkable. The simple diffraction model produces some artifacts at around the shadow boundary (at around $300-400 \mathrm{~m}$ ). This is due to the simple (a) $10 \mathrm{~Hz}$

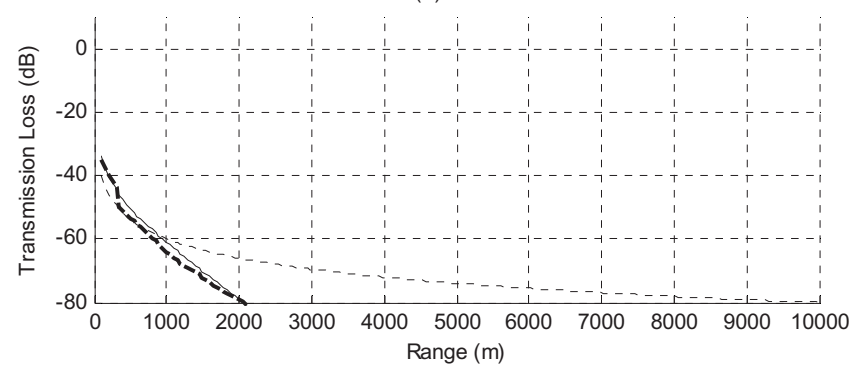

(b) $100 \mathrm{~Hz}$

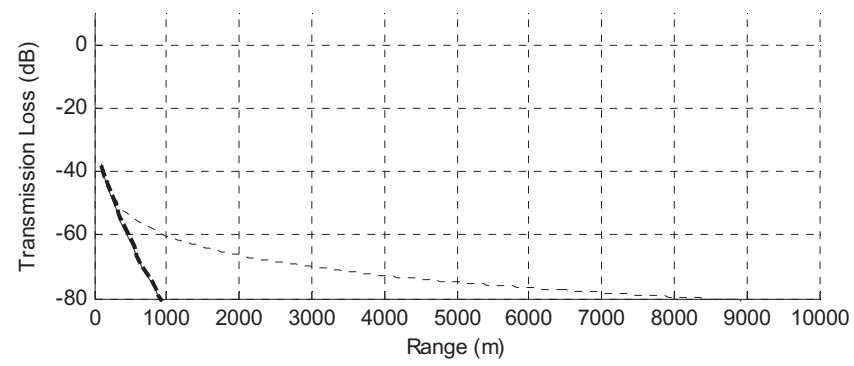

(c) $1000 \mathrm{~Hz}$

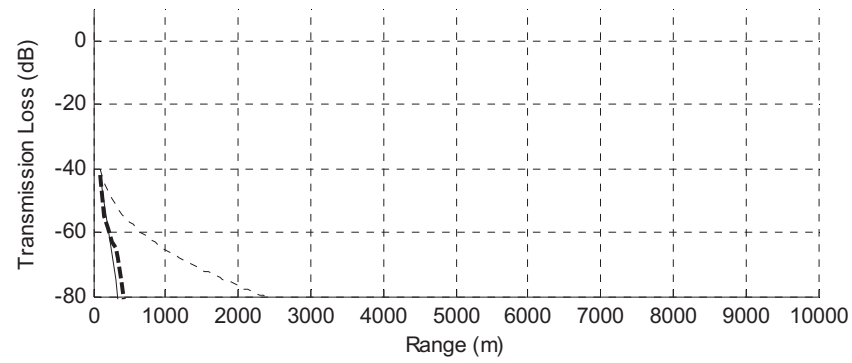

FIG. 2. TL versus distance predicted by the shadow boundary ray diffraction model (broken line) and PE (solid line) for the upward refracting benchmark case 3. The dotted line is for free field in a homogeneous still atmosphere.

ground correction used in the model. The equivalent ground is circular in this benchmark case. Since the ground reflection angle is determined by simple straight lines (see Fig. 1), the angle changes markedly near the shadow boundary (top of the circular arc), thus producing some sudden changes in the sound attenuation. A more elaborate diffraction model that takes into account the ground curvature could improve the accuracy. Nevertheless, the simple model is thought to be close enough to the PE solution to be usable in practice.

\section{TURBULENCE SCATTERING}

The ray based shadow boundary diffraction model of Sec. III provides a convenient basis for extending the calculation to account for the effect of turbulence scattering in the shadow zone. Turbulence can be simulated as temporal and spatial fluctuations of the sound speed along the propagation path. Let the refractive index be $n=c_{0} / c$. $c_{0}$ is a nominal constant sound speed, typically taken as the mean speed on the ground. $c$ is the instantaneous sound speed at the position of interest. The effect of turbulence can be represented as the fluctuating part, $\mu$, of the refractive index at the position of interest, 


$$
n=\bar{n}+\mu,
$$

where $\bar{n}=c_{0} / \bar{c} . \bar{c}$ is the mean sound speed at that position, which is taken to be the value in the absence of turbulence. The fluctuation $\mu$ can be simulated by random realizations, which can be generated based on known models of the turbulence structure. Once $\mu$ is realized, Eq. (1) can be used to calculate the variation in the sound speed gradient due to turbulence within each of the grid cells in the numerical ray tracing algorithm. The ray trajectories will be scattered by these variations. When averaged over a large number of realizations, the result could be used to simulate the scattering of the sound energy by turbulence. For shadow zone calculation, which is the main interest of this paper, the scattering of the shadow boundary ray produces variations of the analogous equivalent diffraction geometry that will in turn change the attenuation detected at the receiver. However, even though ray tracing is faster than other numerical techniques such as the PE solution, performing a complete set of ray tracing for each random realization over a large number of realizations is still too time-consuming for practical use. We will therefore proceed to develop a model that can simulate the scattering of the rays at the receiver without tracing the rays through all the realizations.

\section{A. Sound speed gradient due to turbulence}

Since the numerical ray tracing assumes that the sound speed gradient is linear within each small grid cell in the propagation domain, we will need to relate the change in the gradient to the turbulence fluctuation $\mu$. Rewriting Eq. (1) explicitly in terms of sound speeds, we have

$$
\frac{c}{c_{0}}=\frac{1}{\frac{c_{0}}{\bar{c}}+\mu} \cong \frac{\bar{c}}{c_{0}}\left(1-\frac{\bar{c}}{c_{0}} \mu\right) \text { for } \mu \ll 1 .
$$

The vertical sound speed gradient is given by

$$
\frac{\partial c}{\partial z}=\frac{\partial \bar{c}}{\partial z}-\left(\frac{\bar{c}^{2}}{c_{0}} \frac{\partial \mu}{\partial z}+\frac{2 \mu \bar{c}}{c_{0}} \frac{\partial \bar{c}}{\partial z}\right) \text {. }
$$

In the absence of turbulence, the grid cells are assumed to be small enough that the vertical sound speed gradient within each is approximately linear. Therefore,

$$
\bar{c}=\bar{c}_{1}\left(1+a_{0} z\right)
$$

where $\bar{c}_{1}$ is the mean sound speed at the lower boundary of the cell, and $a_{0}$ is the linear gradient in the absence of turbulence. Generally $\mu$ is small and we may ignore the second term in the parentheses in Eq. (3) compared with the first term in the parentheses. Also, $a_{0}$ and the cell height are small enough such that $\bar{c}^{2} / c_{0} \cong \bar{c}_{1}$. The effect of turbulence is therefore to modify the linear gradient by an extra term. The total gradient, $a$, is then given by

$$
a=\frac{\partial c}{\partial z} \cong a_{0}-\bar{c}_{1} \frac{\partial \mu}{\partial z} .
$$

In the shadow diffraction calculation, the refracting atmosphere is replaced with a still atmosphere with a modified curved ground terrain. In this analogous case, $a_{0}$ and $\bar{c}_{1}$ in Eq. (5) are 0 and $c_{0}$, respectively.

The value of $\mu$ and its derivative can be determined for specific turbulence structures. Here we will use the simple Gaussian turbulence model. ${ }^{18,19}$ Although the Gaussian model is not strictly correct for real atmospheres, it can be adapted to specific frequency ranges to give acceptable results. $^{20}$ Later, we will also show that the Gaussian model provides a convenient basis to simplify the simulation.

The random realization of $\mu(\mathbf{r})$ where the position vector in 2 dimension is $(x, z)$ can be obtained by a spectral decomposition $^{21}$

$$
\mu(\mathbf{r})=\sqrt{4 \pi \Delta k} \sum_{n=1}^{N} \cos \left(\mathbf{k}_{n} \cdot \mathbf{r}+\alpha_{n}\right) \sqrt{F\left(\mathbf{k}_{n}\right) n \Delta k},
$$

where $\mathbf{k}_{n}=\left(k_{n x}, k_{n z}\right)=\left(k_{n} \cos \theta_{n}, k_{n} \sin \theta_{n}\right)$ and $k_{n}=n \Delta k$ for $n$ $=1,2, \ldots, N . N$ is the number of harmonics (or modes) used in the superposition, $\theta_{n}$ and $\alpha_{n}$ are independent random polar angles between 0 and $2 \pi$, and $\Delta k$ is the spectral wavenumber resolution.

For a Gaussian turbulence model, the spectral density function depends on the standard deviations of the temperature and longitudinal wind velocity fluctuations, and the correlation length of the Gaussian spectrum $\ell$. As an approximation, the standard deviation terms are commonly represented by a single $\mu_{0}^{2}$, the square of the standard deviation of the fluctuating part of the index of refraction. We can then write

$$
F\left(k_{x}, k_{z}\right)=\mu_{0}^{2} \frac{\ell^{2}}{4 \pi} e^{-\left(k_{x}^{2}+k_{z}^{2}\right) \ell^{2} / 4} .
$$

The derivative of $\mu(\mathbf{r})$ is then

$$
\frac{\partial \mu(\mathbf{r})}{\partial z}=-\sqrt{4 \pi \Delta k} \sum_{n=1}^{N} k_{n z} \sin \left(\mathbf{k}_{n} \cdot \mathbf{r}+\alpha_{n}\right) \sqrt{F\left(\mathbf{k}_{n}\right) n \Delta k} .
$$

This equation can be used to determine the sound speed gradient within a grid cell in the presence of turbulence according to Eq. (5).

\section{B. Turbulence scattered rays}

In the presence of turbulence, the shadow boundary ray is scattered as it propagates through the atmosphere. This is illustrated in the upper part of Fig. 1. Each random realization will produce a different scattered path. In principle, an analogous equivalent curved ground can be used to calculate the diffraction from each of this scattered boundary ray. However, this will be very time-consuming since a complete ray tracing will have to be done for each realization. Instead, we note that the dominant effect on the diffraction amplitude is due to the change in the height, denoted by $h_{r}$ in Fig. 1, between the shadow boundary and the receiver. Hence our objective is to find a simple way to simulate this change in height due to turbulence.

Consider the propagation of a ray through a randomly realized turbulent atmosphere. The ray is traced numerically through a series of grid cells within each the sound speed gradient is approximately linear, as calculated by Eq. (5) in 
the presence of turbulence. Note that the grid cell size in this case is set at $1 / 10$ of the Gaussian correlation length $\ell$ to make sure that the randomly realized refraction index fluctuations are properly sampled.

It can be shown ${ }^{8}$ analytically that the horizontal angle at which the ray enters the cell, $\phi_{1}$, is related to the angle at which the ray exits the cell, $\phi_{2}$, by the following equations:

$$
\sin \phi_{2}=\sin \phi_{1}-\frac{x_{2}-x_{1}}{R}
$$

and

$$
\cos \phi_{2}=\cos \phi_{1}+\frac{z_{2}-z_{1}}{R},
$$

where $\left(x_{1}, z_{1}\right)$ and $\left(x_{2}, z_{2}\right)$ are, respectively, the coordinates of the entry and exit points. $R$ is the radius of the ray curvature given by

$$
R=\frac{1}{a \cos \phi_{1}} .
$$

The change in height, $\Delta z=z_{2}-z_{1}$, after the ray passes through this cell, is then

$$
\Delta z=R\left(\cos \phi_{2}-\cos \phi_{1}\right) .
$$

Now, let us consider the analogous curved ground diffraction model, in which the shadow boundary ray is equivalent to a straight ray in the absence of turbulence. In this model, the effective linear sound speed gradient in each of the grid cells is created by the turbulence alone, i.e., $a_{0}=0$ in Eq. (5), and is the sole cause of the scattering and the change in ray height. The ray horizontal angle will be small, especially in long range propagation, and we can approximate Eqs. (12) and (9) by

$$
\Delta z \cong R\left(\frac{\phi_{1}^{2}-\phi_{2}^{2}}{2}\right) \cong \Delta x\left(\frac{\phi_{2}+\phi_{1}}{2}\right),
$$

where $\Delta x=x_{2}-x_{1}$. In the numerical ray tracing procedure, each cell has the same length $\Delta x$. For the $i$ th cell, and for small horizontal angles, Eq. (9) gives

$$
\phi_{i+1} \cong \phi_{i}-\frac{\Delta x}{R_{i}}
$$

Note that the entry horizontal angle into cell $i$ is the same as the exit horizontal angle from cell $i-1$. Applying this formula recursively, and taking the initial horizontal angle of the ray at source to be $\phi_{0}$, we have

$$
\phi_{i+1} \cong \phi_{0}-\sum_{j=1}^{i} \frac{\Delta x}{R_{j}} .
$$

Substituting this into Eq. (13) for the $i$ th cell gives the cumulative height change at the exit of cell $i$ to be

$$
\begin{aligned}
\Delta z_{i+1} & \cong \Delta x \frac{\left(\phi_{i+1}+\phi_{i}\right)}{2} \\
& \cong \phi_{0}+\frac{\Delta x}{2}\left(\sum_{j=1}^{i} \frac{-\Delta x}{R_{j}}+\sum_{j=1}^{i-1} \frac{-\Delta x}{R_{j}}\right) \\
& =\phi_{0}-\Delta x^{2}\left(\sum_{j=1}^{i} \frac{1}{R_{j}}-\frac{1}{2 R_{i}}\right) .
\end{aligned}
$$

Again, taking the assumption that the horizontal angles are small, we can approximate Eq. (11) by $R_{j} \approx 1 / a_{j}$. Equation (16) then becomes

$$
\Delta z_{i+1} \cong \phi_{0}-\Delta x^{2}\left(\sum_{j=1}^{i} a_{j}-\frac{a_{i}}{2}\right) .
$$

The gradient $a_{i}$ is different for each cell. However, for a homogenous turbulence structure, all the $a_{i}$ 's are determined from the same turbulence model, Eqs. (5) and (8). They are all proportional to the turbulence strength represented by the square root of $\mu_{0}^{2}$, i.e., the square of the standard deviation of the fluctuating part of the index of refraction for a Gaussian turbulence model. Hence we can write

$$
a_{j}=q_{j} \sqrt{\mu_{0}^{2}},
$$

where the proportional factor $q_{j}$ is determined from Eq. (8) for each cell. We can now rewrite Eq. (17) as

$$
\Delta z_{i+1} \cong \phi_{0}-\sqrt{\mu_{0}^{2}} \Delta x^{2}\left(\sum_{j=1}^{i} q_{j}-\frac{q_{i}}{2}\right) .
$$

This equation is independent of frequency. Moreover, all the variables in the equation except $\mu_{0}^{2}$ are independent of the turbulence strength. For long range propagation, and for the analogous shadow boundary diffraction model, the initial horizontal ray angle $\phi_{0}$ is small and close to zero. This means that the ray tracing simulation needs only be done once, for one turbulence strength, and the result can be applied to other turbulence strength through a simple scaling. Although the factor $q_{j}$ is dependent on the Gaussian correlation length $\ell$, in practice, $\ell$ is generally taken as a constant $1.1 \mathrm{~m}$ in a Gaussian model.

\section{Changes in shadow ray height due to turbulence}

Since we are using a Gaussian turbulence model to generate the random realizations, we expect the resulting change in height $(\Delta z)$ to exhibit Gaussian behavior. Figure 3 shows the result of a simulation of the scattering of a horizontal ray through a Gaussian turbulence atmosphere with $\mu_{0}^{2}$ set to $3 \times 10^{-6}$. The correlation length $\ell$ is set to $1.1 \mathrm{~m}$ in this and all subsequent calculations. The result is from 100 random realizations of the turbulent atmosphere. The size of each grid cell is set at $0.11 \mathrm{~m}$, which is $1 / 10$ of the Gaussian correlation length $\ell$ to make sure that the randomly realized refraction index fluctuations are properly sampled. The changes in the ray height due to turbulence scattering at a distance of $1 \mathrm{~km}$ were recorded. The cumulative probability distribution function (CDF) of the height change is plotted in the figure. It can be seen that the CDF looks similar to that of 


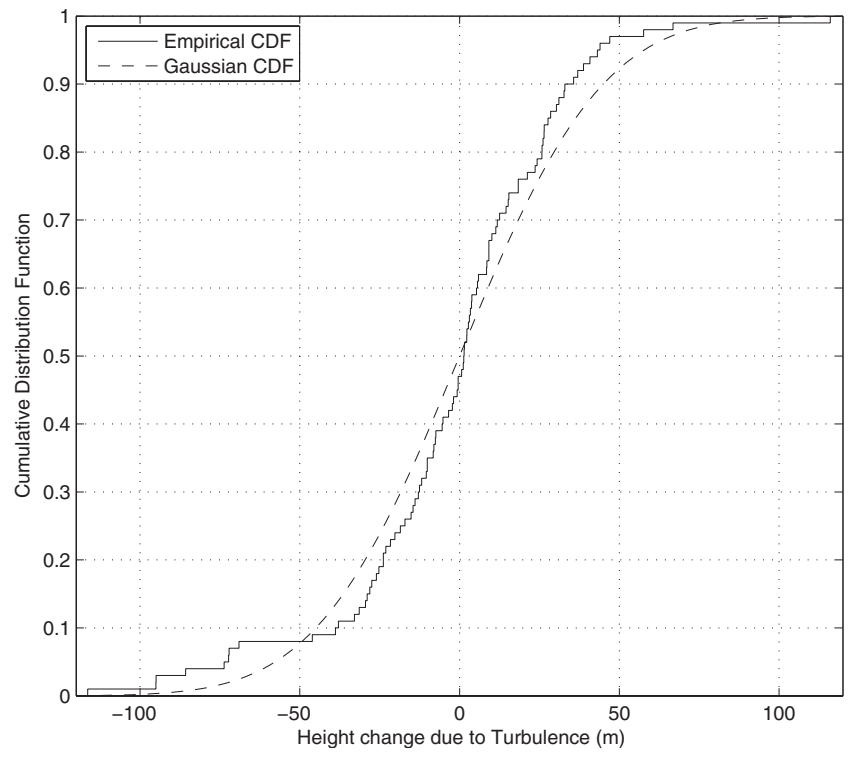

FIG. 3. The CDF of the simulated height change (solid line) of a horizontal ray after propagated $1 \mathrm{~km}$ through an atmosphere with a Gaussian turbulence structure using 100 random realizations. The dotted line is that of a standard Gaussian distribution.

an ideal Gaussian distribution of zero mean. From here on, we assume that we could take the distribution of height change $(\Delta z)$ in Eq. (19) over many random realizations to be Gaussian with zero mean, and can be characterized by its standard deviation.

We now need to determine the dependence of $\Delta z$ on range. This comes out from the term in parentheses on the right hand side of Eq. (19). Again, the propagation of a horizontal ray through 100 random realizations was used to simulate the height changes due to turbulence. The turbulence model is again Gaussian, with $\mu_{0}^{2}$ set at $3 \times 10^{-6}$. The values of the standard deviation of the height change at every $20 \mathrm{~m}$ up to $10 \mathrm{~km}$ were calculated from the simulated realizations. Note that $0 \mathrm{~m}$ corresponds to the start of the shadow zone. Figure 4 shows the result. The standard deviation's dependency with range is smooth, and is almost linear on a $\log -\log$ scale. The dependency is almost linear at short to medium range up to $1 \mathrm{~km}$. At longer range the standard deviation grows faster and a higher order curve is needed. It is

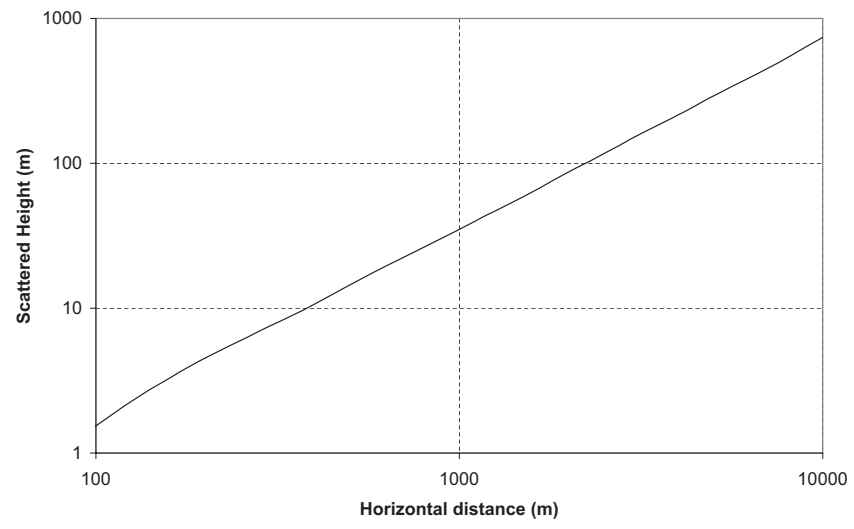

FIG. 4. Dependence of the standard deviation of the scattered height statistics with range. The turbulence model used to generate the 100 random realizations is the same as that of Fig. 3 .
TABLE I. Coefficients for the estimation of the standard deviation of height changes in Eq. (20).

\begin{tabular}{lllll}
\hline \hline Coefficient & $a_{11}$ & $a_{31}$ & $a_{32}$ & $a_{33}$ \\
Value & 0.13 & 0.10 & $3.2 \times 10^{-5}$ & $-1.2 \times 10^{-9}$ \\
\hline \hline
\end{tabular}

apparent that, up to the range of $10 \mathrm{~km}$, the dependency on range can be represented easily by simple regression curves. This is a useful result since a simple regression curve would allow us to calculate the standard deviation at any range without needing to perform time-consuming ray tracing through many random realizations again. Note that the size of each grid cell in the numerical ray tracing is again set at $0.11 \mathrm{~m}, 1 / 10$ of the Gaussian correlation length $\ell$, in order to sample the turbulence properly. Since the maximum range in the simulation is $10 \mathrm{~km}$, this requires calculation of ray refractions over approximately 91000 cells for each random realization. This is obviously time-consuming, and the ability to replace this with a simple regression formula is significant.

As a simple choice, we used a linear regression for the range from 0 to $1 \mathrm{~km}$, and then a third order regression from 1 to $10 \mathrm{~km}$. The resulting coefficients are listed in Table I. An equation for the standard deviation of the height change, $\Delta z_{\mathrm{STD}}$, for near horizontal propagation $\left(\phi_{0} \approx 0\right)$ can now be written as

$$
\begin{aligned}
\Delta z_{\mathrm{STD}}(x) & =\sqrt{\frac{\mu_{0}^{2}}{\mu_{\mathrm{ref}}^{2}}} a_{11} x \quad \text { for } 0<x \leq 1 \mathrm{~km} \\
& =\sqrt{\frac{\mu_{0}^{2}}{\mu_{\mathrm{ref}}^{2}}}\left(a_{31} x+a_{32} x^{2}+a_{33} x^{3}\right) \text { for } x>1 \mathrm{~km} .
\end{aligned}
$$

The scaling, with respect to the turbulence strength, is taken from Eq. (19). The reference turbulence strength is set at $\mu_{\text {ref }}^{2}=3 \times 10^{-5}$ to give more convenient values to the coefficients.

Equations (19) and (20) predict that $\Delta z$ can be described by a Gaussian distribution and that it is proportional to $\sqrt{\mu_{0}^{2}}$. To test the dependence of the height changes on the strength of turbulence, ray tracing simulations were repeated over a range of $\mu_{0}^{2}$ values from $1 \times 10^{-6}$ to $7 \times 10^{-5}$. The standard deviations of the height changes at a number of distances from the shadow boundary are plotted against the square root of $\mu_{0}^{2}$ in Fig. 5. It can be seen that the standard deviation does indeed scale very well with $\sqrt{\mu_{0}^{2}}$ at all the ranges. Also shown in the graph as dotted lines are the standard deviations that are calculated simply from Eq. (20). The prediction from the simple formula matches fairly well with the simulated result from the full numerical ray tracing. The error is largely less than $10 \%$. In the $500 \mathrm{~m}$ case where the height change is small the error can go up to $30 \%$. There is also a tendency for the simulated standard deviation to be slightly larger than that from Eq. (20) at the extremes: when the range is $>2 \mathrm{~km}$ (very deep in shadow) and the turbulence strength is very high $\left(>5 \times 10^{-5}\right)$. Overall, the simple equation is a good replacement of the time-consuming ray tracing simulation under realistic conditions. 


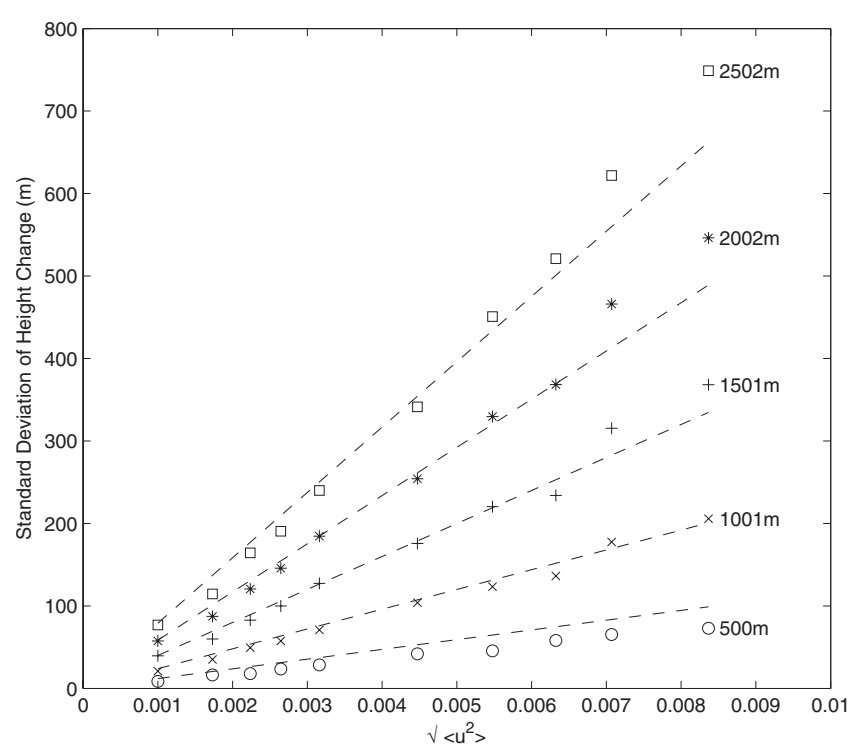

FIG. 5. Variation of the standard deviation of the simulated height changes against the square root of $\mu_{0}^{2}$ of the Gaussian type turbulence. The symbols are results from numerical ray tracing using 100 random realizations for each turbulence strength. The dashed lines are direct calculations from the approximate formula.

\section{TURBULENCE SCATTERED SHADOW RAY (TSSR) MODEL}

With the results from Sec. IV, we can now calculate the shadow zone sound pressure in the presence of turbulence by the following steps.

(1) The limiting ray that defines the shadow boundary in an upward refracting atmosphere is first determined by a single numerical ray tracing process in the absence of turbulence.

(2) The shadow boundary geometry is replaced with an equivalent configuration of a straight horizontal ray passing just above the top of a curved ground with geometry defined by the original curve shadow boundary ray, as shown in Fig. 1. This horizontal ray effectively defines the line of sight in the modified ground geometry. In the absence of turbulence, the sound pressure level in the shadow zone is simply calculated as a barrier diffraction problem with an impedance ground.

(3) In the presence of turbulence, the turbulence structure is modeled as Gaussian, with the strength represented by $\mu_{0}^{2}$. The correlation length of the Gaussian spectrum $\ell$ is assumed to be fixed and equal to $1.1 \mathrm{~m}$. The effect of turbulence is to be represented by the scattering of the ray height above the receiver.

(4) Equation (20) allows the standard deviation of the ray height changes, $\Delta z_{\mathrm{STD}}$, due to turbulence to be calculated at any range up to $10 \mathrm{~km}$ from the shadow boundary, and for any turbulence strength $\mu_{0}^{2}$.

(5) $\Delta z_{\text {STD }}$ is then used to generate random height changes from a Gaussian distribution with zero mean. A positive height change increases the height of the ray and therefore extends the height separation between the receiver and the line of sight. This will then increase the attenuation due to diffraction. Conversely, a negative height
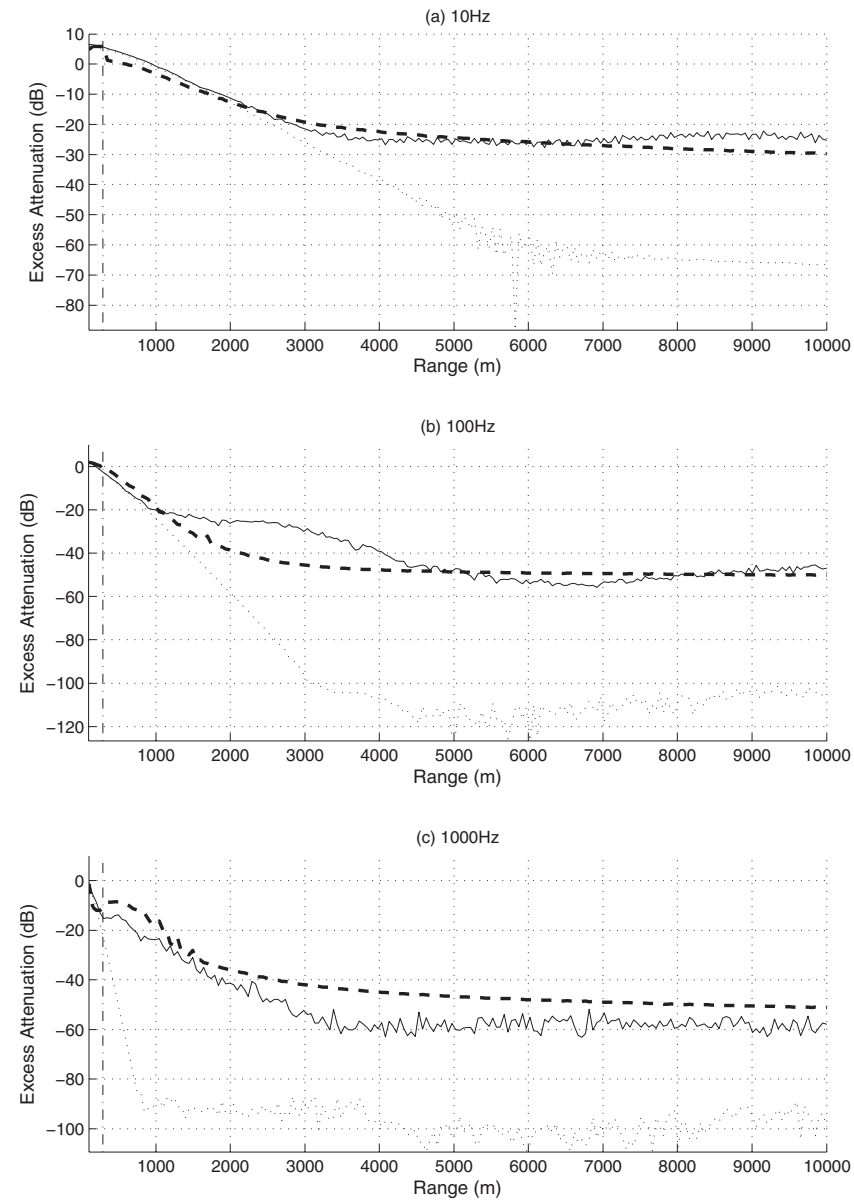

FIG. 6. Excess attenuation versus distance predicted by the TSSR model (broken line) and PE (solid line) for the modified upward refracting benchmark case 3 with turbulence added. A Gaussian turbulence model is assumed with a strength of $\mu_{0}^{2}=3 \times 10^{-6}$. The dotted line is the PE prediction for no turbulence. The vertical chained line indicates the estimated shadow boundary at $300 \mathrm{~m}$.

change decreases the height of the ray and therefore reduces the height separation between the receiver and the line of sight. The will then decrease the attenuation due to diffraction. With each height change, a new diffracted sound pressure level is calculated from the modified diffraction geometry.

(6) Finally, the attenuation values calculated from all the realizations are energy averaged to give the mean attenuation due to shadowing in the presence of turbulence.

For simplicity, we abbreviate this procedure as the TSSR model. To test the accuracy of the model, it was used to calculate the sound pressure level in the shadow zone of benchmark case 3 with the addition of turbulence. Since the published benchmark case result does not include turbulence, it is necessary to compare the result with a numerical calculation that has established accuracy. In this case, the standard PE solution with the addition of the randomization procedure of Gilbert et al. ${ }^{1}$ for the simulation of turbulence is used as the reference. The same Gaussian turbulence structure is used in both the PE calculation and the ray tracing calculation. The result for a moderate turbulence strength of $\mu_{0}^{2}=3$ $\times 10^{-6}$ is shown in Fig. 6 . The PE prediction with no turbu- 


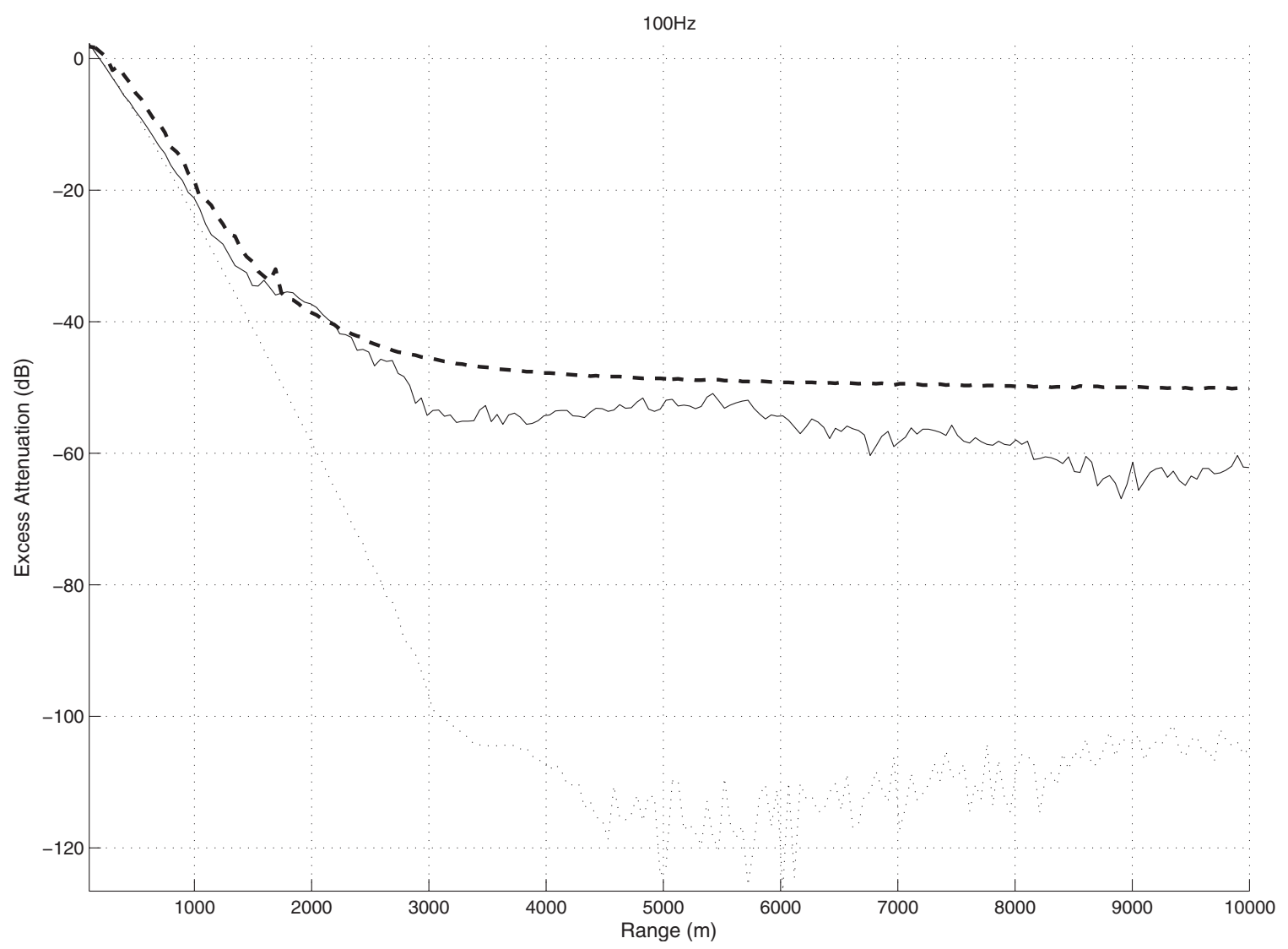

FIG. 7. Same as Fig. 6(b) but with a Gaussian correlation length of $4 \mathrm{~m}$.

lence is also shown in the figure to let the effect of turbulence to be seen clearly. As mentioned before, this benchmark case is strongly upward refracting, with the shadow boundary starts at about $300 \mathrm{~m}$ from the source, as shown by the vertical chained line in the figure. Most of the range shown in the figure is therefore in the shadow zone. At the lowest frequency, $10 \mathrm{~Hz}$, a sharp transition can be seen at the shadow boundary at around $300 \mathrm{~m}$ where the calculation changes from the normal ray tracing calculation before the shadow boundary to the TSSR calculation beyond the shadow boundary. The sharp change is of the order of $5 \mathrm{~dB}$. This change can be easily explained. Before the shadow, the ground reflection detected by the ray tracing will be largely in phase with the direct sound at this low frequency. Hence the sound level increases by around $6 \mathrm{~dB}$ due to the ground. Immediately behind the shadow boundary, the calculation switches to the TSSR model since no rays arrive. Right at the boundary, the diffracted ray is at line of sight between source and the receiver. The attenuation given by the geometrical diffraction model at line of sight is about $5 \mathrm{~dB}$. Hence a sudden increase in attenuation of about $5 \mathrm{~dB}$ is seen when the calculation switches to the TSSR model at the shadow boundary. Note that the $6 \mathrm{~dB}$ pressure level increase due to ground reflection is still retained as the TSSR model also accounts for the reflection by the ground behind the shadow boundary. Hence the drop is only $5 \mathrm{~dB}$. If the ground effect is not included in the calculation behind the shadow, an $11 \mathrm{~dB}$ difference would have been seen. This shows the importance of accounting for the ground effect even in the shadow zone in this model. In principle, this sudden change can be avoided if the effect of diffraction is introduced gradually before the receiver hits the shadow boundary. However, since the ray tracing model is not aimed at low frequency applications, we have not made such adjustments in our current model. Further into the shadow, the TSSR prediction agrees very well with the PE prediction.

At the higher frequencies, $100 \mathrm{~Hz}$ and $1 \mathrm{kHz}$, the sudden change at the shadow boundary is no longer observable. This is because the ground reflection is no longer in phase with the direct sound at higher frequencies, and attenuation starts to appear even before the shadow. At $100 \mathrm{~Hz}$, the TSSR prediction matches the PE prediction closely all the way up to about $1 \mathrm{~km}$. From then on, the PE result shows an unexpected increase in level at ranges up to $4 \mathrm{~km}$, then a slight decrease afterwards. This variation resembles some form of interference effect. Further testing of the PE suggests that this is related to the combination of the specific $100 \mathrm{~Hz}$ frequency and the particular Gaussian turbulence parameters used, and is exaggerated by the extremely large sound speed gradient of the benchmark case. For example, changing the Gaussian length scale from 1.1 to $4 \mathrm{~m}$ changes the shape notably, as shown in Fig. 7. Also, as shown later in Fig. 8, this spurious increase is not seen when a higher turbulence strength is used. Further investigation into this spurious behavior of the PE in combination with a Gaussian turbulence model is, however, outside the scope of this paper, although it could be an interesting subject for future work. Otherwise the overall trends of the excessive attenuation predicted by the two models appear to agree. 

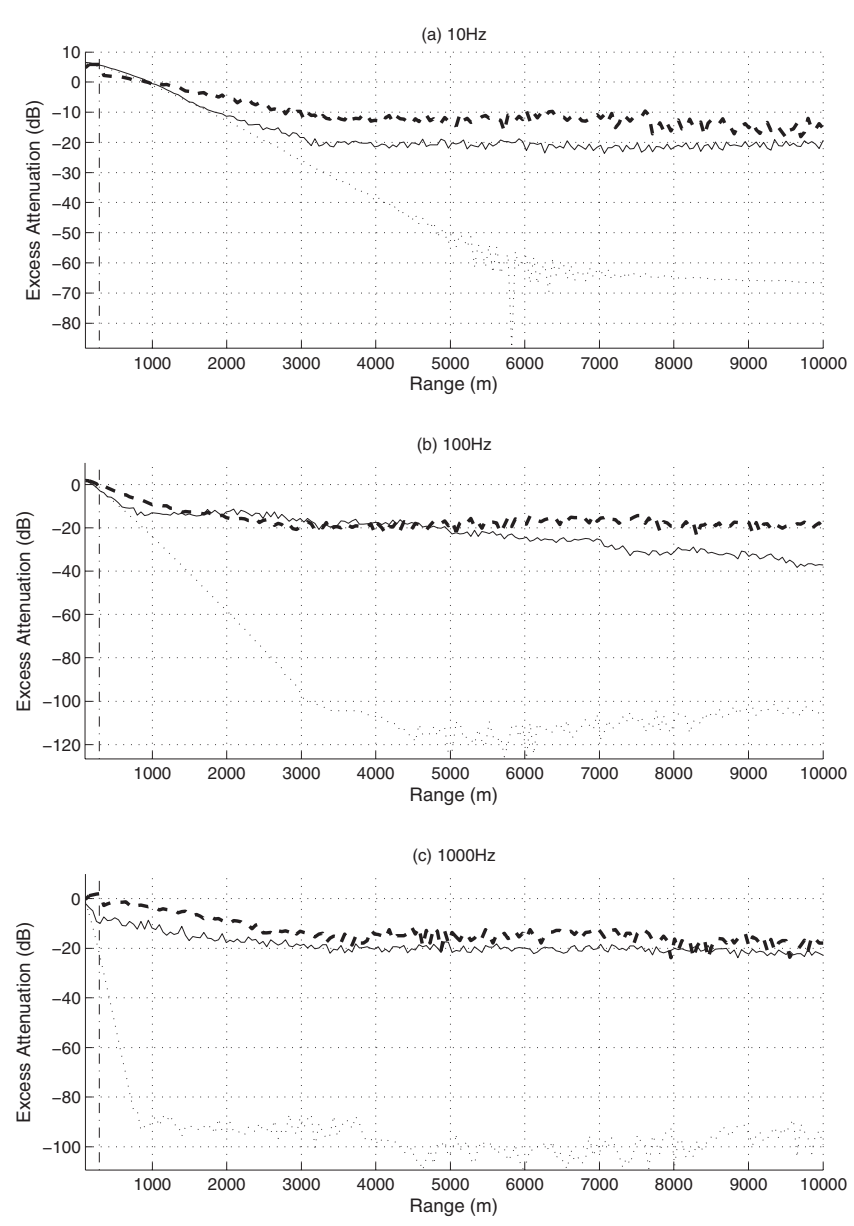

FIG. 8. Same as Fig. 6 but with $\mu_{0}^{2}=3 \times 10^{-5}$.

At $1 \mathrm{kHz}$, the TSSR predicts consistently smaller magnitude of attenuation compared with the PE prediction. The difference is around $5 \mathrm{~dB}$ up to $2 \mathrm{~km}$, and increases to around $10 \mathrm{~dB}$ or more at longer ranges.

The effect of larger turbulence is shown in Fig. 8, where the turbulence strength is at a high level of $\mu_{0}^{2}=3 \times 10^{-5}$. This is considered to be the upper limit of turbulence that one would normally encounter. With this high level of turbulence, the attenuation in the shadow is severely limited, reaching just over -10 to $-20 \mathrm{~dB}$ at $10 \mathrm{~Hz}$, and around $-20 \mathrm{~dB}$ at the higher frequencies even at $10 \mathrm{~km}$ into the shadow. At $10 \mathrm{~Hz}$, the TSSR prediction is still fairly close to that of the PE model, but is consistently less than the PE attenuation from about $1 \mathrm{~km}$ onwards. At $100 \mathrm{~Hz}$, the agreement is very good in the region up to about $5 \mathrm{~km}$. The spurious increase in sound level between 1 and $4 \mathrm{~km}$, seen earlier in the PE result in Fig. 6(b), at this frequency does not appear at this higher level of turbulence. From $5 \mathrm{~km}$ onwards, the TSSR predicted attenuation is again consistently less than that from the PE. A similar trend is also observed in the $1 \mathrm{kHz}$ result. Overall, the results show that the TSSR prediction has good agreement with the PE prediction at short to medium range from the shadow boundary at all the test frequencies. At ranges further than $3.5 \mathrm{~km}$, the TSSR predicts smaller attenuation values than the PE model.

As a further test of the reliability of the TSSR model, predictions were made and compared against the measured
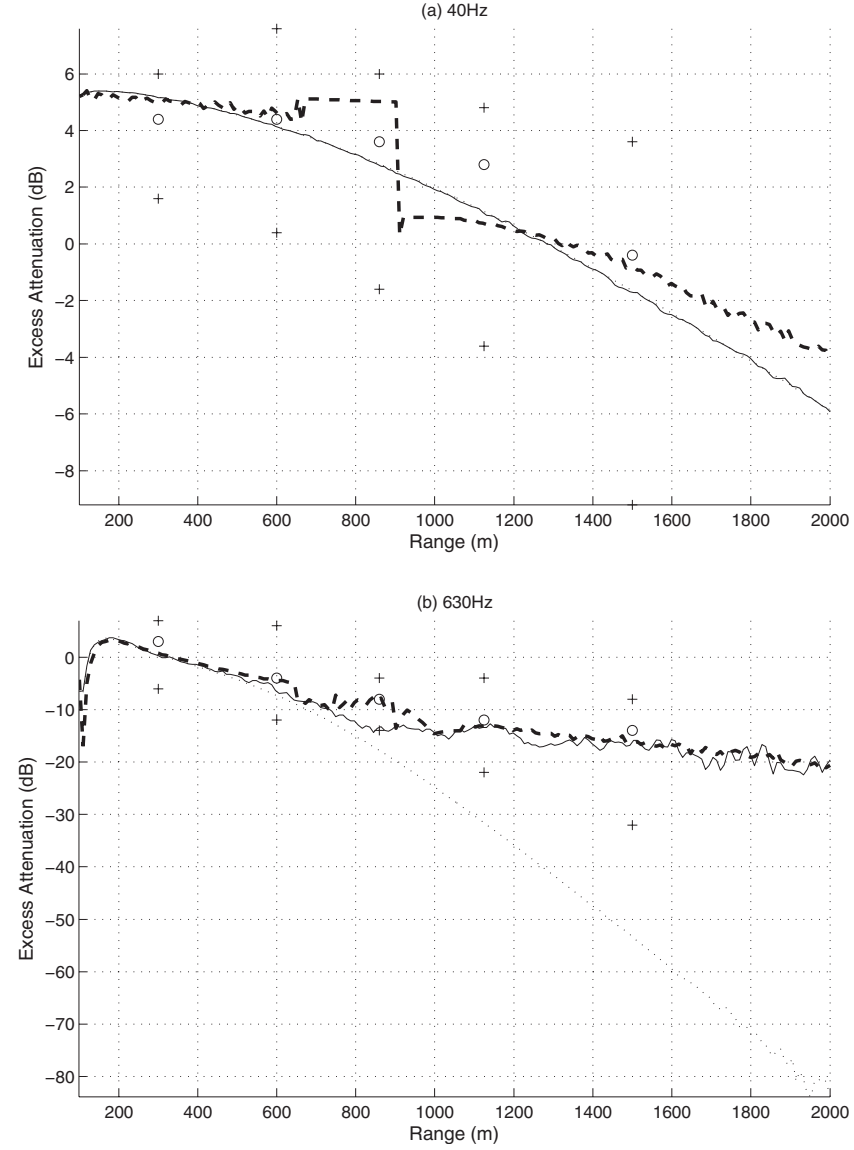

FIG. 9. Excess attenuation versus distance predicted by the TSSR model (thick broken line) and PE (solid line) against experimental data published by White and Gilbert (Ref. 22). The assumed sound speed gradient is $-0.034 / \mathrm{s}$, and $\mu_{0}^{2}=1 \times 10^{-6} .+, \bigcirc$, and + represent the maximum, mean, and minimum experimental results of White and Gilbert (Ref. 22).

data of White and Gilbert. ${ }^{22}$ These data had been used by L'Espérance et al. ${ }^{8}$ in their development of a heuristic ray tracing model, although their comparison was not entirely successful due to the absence of a proper turbulence model in the shadow zone. Since the purpose of this paper is on the diffraction and turbulence effect in the shadow zone, only the upward refracting data are included in this comparison. The meteorological parameters used are the estimated values used by L'Espérance et al. ${ }^{8}$ (see Figs. 11 and 12 of Ref. 8). The estimated linear sound speed gradients for the two upward refracting cases are $-0.034 / \mathrm{s}$ and $-0.068 / \mathrm{s}$. The corresponding values of $\mu_{0}^{2}$ for the approximate Gaussian turbulence are $1 \times 10^{-6}$ and $2 \times 10^{-6}$, respectively. The data set includes two frequencies-a low frequency of $40 \mathrm{~Hz}$ and a medium frequency of $630 \mathrm{~Hz}$. The two cases are shown in Figs. 9 and 10. In both cases the TSSR model predictions agree well with the measured data, except at the shadow boundary at $40 \mathrm{~Hz}$ where a sharp change of about $5 \mathrm{~dB}$ is seen. This sharp change is due to the switch over at the boundary from a hard ground effect at low frequency to a diffraction calculation in the TSSR model. This has already been discussed in detail earlier. It is interesting to note that even with this sharp change, the predicted values are still within the minimum to maximum range of the measured values. This error is not seen at the higher frequency, 

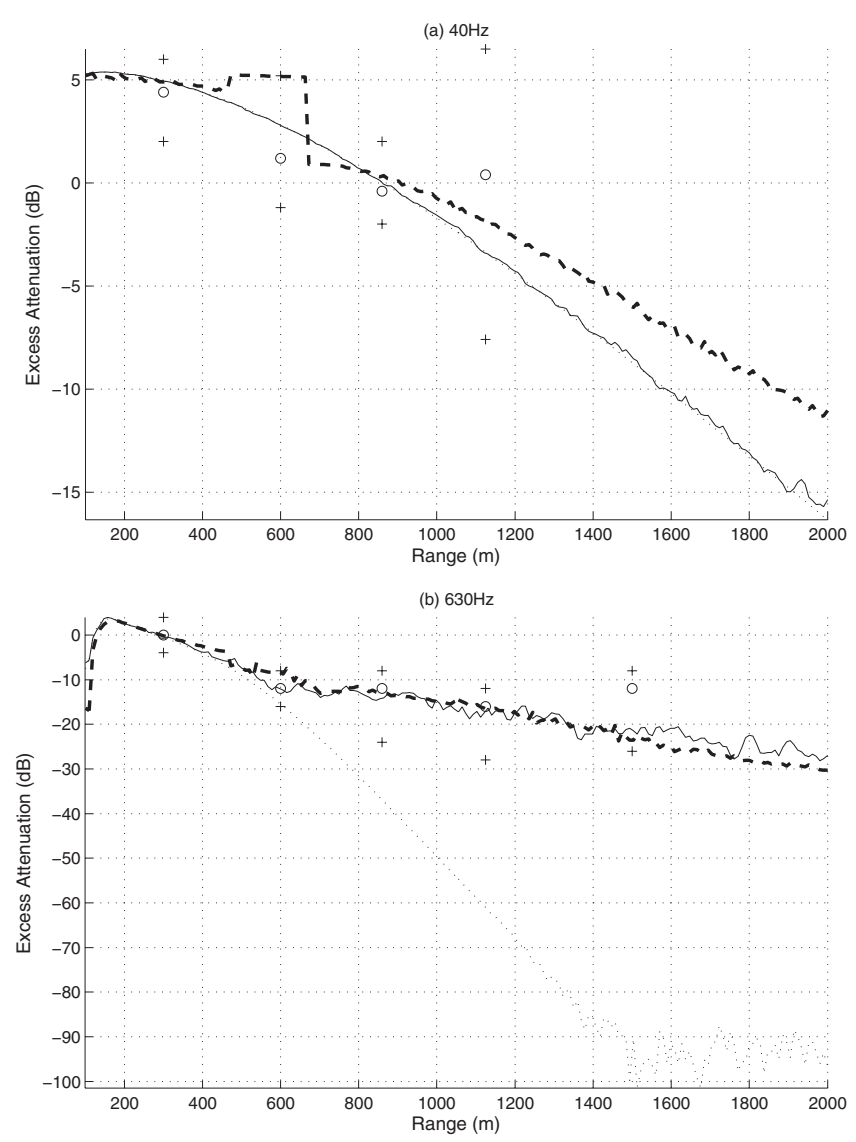

FIG. 10. Same as Fig. 9 but with sound speed gradient of $-0.068 / \mathrm{s}$, and $\mu_{0}^{2}=2 \times 10^{-6}$.

$630 \mathrm{~Hz}$, where the agreement is very good. The agreement between the TSSR model and the PE model in these realistic cases is significantly better than that observed in the extreme benchmark cases shown in Figs. 6 and 8 within the corresponding horizontal ranges.

\section{CONCLUSIONS}

In this paper, a simple analytical model has been developed for the scattering of rays due to turbulence in a shadow zone. The model is based on the assumption of a Gaussian turbulence structure, and makes use of the equivalence of ray propagation in a refractive atmosphere over flat ground and the propagation in a still atmosphere over a curved ground. The equations are derived analytically in all but one aspect. The equation for the standard deviation of the changes in ray height due to turbulence is obtained from statistics generated by numerical simulations. Nevertheless, this equation is shown to be valid for a horizontal distance of $10 \mathrm{~km}$ over a large range of turbulence strengths for an assumed Gaussian turbulence structure.

This simple analytical model has been tested in a simulated benchmark case and against previously published measured data at a variety of frequencies and turbulence strengths. The excess attenuation predicted by the model in the shadow zone has been shown to agree well with that predicted by a standard PE solution in all the test cases at distances up to $2 \mathrm{~km}$ from the shadow boundary. Right at the shadow boundary, there is an error of up to $5 \mathrm{~dB}$ at low frequency where the ground is effectively hard. This is due to the step change of attenuation from hard ground pressure doubling to a line-of-sight barrier screening effect in the model. This error does not appear at frequencies higher than $100 \mathrm{~Hz}$ in the test cases. Further into the shadow zone, from 2 to $10 \mathrm{~km}$, the model tends to predict less excess attenuation than the PE.

The comparisons presented in this paper have shown that the TSSR model provides fairly accurate predictions of excess attenuation in the shadow zone at distances up to $2 \mathrm{~km}$ from the boundary under a variety of turbulence strengths. The formulation of the model is based on ray tracing, and is therefore well suited to such geometrical models, which up to now have problems dealing with attenuation in the shadow zone. The model can also be used as a simple, standalone prediction tool for the attenuation in a shadow zone due to turbulence.

${ }^{1}$ K. E. Gilbert, R. Raspet, and X. Di, "Calculation of turbulence effects in an upward-refracting atmosphere," J. Acoust. Soc. Am. 87, 2428-2437 (1990).

${ }^{2}$ W. E. McBride, H. E. Bass, R. Raspet, and K. E. Gilbert, "Scattering of sound by atmospheric turbulence: Predictions in a refractive shadow zone," J. Acoust. Soc. Am. 91, 1336-1340 (1992).

${ }^{3}$ A. L'Espérance, Y. Gabillet, and G. A. Daigle, "Outdoor sound propagation in the presence of atmospheric turbulence: Experiments and theoretical analysis with the fast field program algorithm," J. Acoust. Soc. Am. 98, 570-579 (1995).

${ }^{4}$ K. E. Gilbert and X. Di, "A fast Green's function method for one-way sound propagation in the atmosphere," J. Acoust. Soc. Am. 94, 2343-2352 (1993).

${ }^{5}$ A. D. Pierce, Acoustics: An Introduction to Its Physical Principles and Applications (McGraw-Hill, New York, 1981), pp. 371-413 and 469-478.

${ }^{6} \mathrm{E}$. M. Salomons, "Downwind propagation of sound in an atmosphere with a realistic sound-speed profile: A semianalytical ray model," J. Acoust. Soc. Am. 95, 2425-2436 (1994).

${ }^{7}$ M. M. Boone and E. A. Vermaas, "A new ray-tracing algorithm for arbitrary inhomogeneous and moving media including caustics," J. Acoust. Soc. Am. 90, 2109-2117 (1991).

${ }^{8}$ A. L'Espérance, P. Herzog, G. A. Daigle, and J. R. Nicolas, "Heuristic model for outdoor sound propagation based on an extension of the geometrical ray theory in the case of linear sound speed profile," Appl. Acoust. 37, 111-139 (1992).

${ }^{9}$ D. Heimann and G. Gross, "Coupled simulation of meteorological parameters and sound level in a narrow valley," Appl. Acoust. 56, 73-100 (1999).

${ }^{10} \mathrm{R}$. Raspet, G. E. Baird, and W. Wu, "The relationship between upward refraction above a complex impedance plane and the spherical wave evaluation for a homogeneous atmosphere," J. Acoust. Soc. Am. 89, 107114 (1991).

${ }^{11}$ J. Hofmann and K. Heutschi, "An engineering model for sound pressure in shadow zones based on numerical simulations," Acta Acust. 91, 661-670 (2005).

${ }^{12}$ Y. W. Lam and M. R. Monazzam, "On the modeling of sound propagation over multi-impedance discontinuities using a semiempirical diffraction formulation," J. Acoust. Soc. Am. 120, 686-698 (2006).

${ }^{13} \mathrm{C}$. F. Chien and W. W. Soroka, "Sound propagation along an impedance plane," J. Sound Vib. 43, 9-20 (1975).

${ }^{14}$ A. Berry and G. A. Daigle, "Controlled experiments on the diffraction of sound by a curved surface," J. Acoust. Soc. Am. 83, 2047-2058 (1988).

${ }^{15}$ K. E. Gilbert and M. J. White, "Application of the parabolic equation to sound propagation in a refracting atmosphere," J. Acoust. Soc. Am. 85, 630-637 (1989).

${ }^{16}$ R. A. Sack and M. West, "A parabolic equation for sound propagation in two dimensions over any smooth terrain profile: The generalised terrain parabolic equation (GT-PE)," Appl. Acoust. 45, 113-129 (1995).

${ }^{17}$ K. Attenborough, S. Taherzadeh, H. E. Bass, X. Di, R. Raspet, G. R. Becker, A. Güdesen, A. Chrestman, G. A. Daigle, A. L'Espérance, Y. Gabillet, K. E. Gilbert, Y. L. Li, M. J. White, P. Naz, J. M. Noble, and H. A. J. M. van Hoof, "Benchmark cases for outdoor sound propagation 
models," J. Acoust. Soc. Am. 97, 173-191 (1995).

${ }^{18}$ G. A. Daigle, J. E. Piercy, and T. F. W. Embleton, "Effects of atmospheric turbulence on the interference of sound waves near a hard boundary," J. Acoust. Soc. Am. 64, 622-630 (1978).

${ }^{19}$ G. A. Daigle, J. E. Piercy, and T. F. W. Embleton, "Line-of-sight propagation through atmospheric turbulence near the ground," J. Acoust. Soc Am. 74, 1505-1513 (1983).
${ }^{20}$ D. K. Wilson, J. G. Brasseur, and K. E. Gilbert, "Acoustic scattering and the spectrum of atmospheric turbulence," J. Acoust. Soc. Am. 105, 30-34 (1999).

${ }^{21}$ E. M. Salomons, Computational Atmospheric Acoustics (Kluwer, Dordrecht, 2001), pp. 215-227.

${ }^{22}$ M. J. White and K. E. Gilbert, "Application of the parabolic equation to the outdoor propagation of sound," Appl. Acoust. 27, 227-238 (1989). 